\title{
Recoupling the singlet- and triplet-pairing channels in single-reference coupled cluster theory
}

\author{
John A. Gomez, ${ }^{1}$ Thomas M. Henderson, ${ }^{1,2}$ and Gustavo E. Scuseria ${ }^{1,2}$ \\ ${ }^{1}$ Department of Chemistry, Rice University, Houston, Texas 77005, USA \\ ${ }^{2}$ Department of Physics and Astronomy, Rice University, Houston, Texas 77005, USA
}

(Received 5 August 2016; accepted 20 September 2016; published online 4 October 2016)

\begin{abstract}
It is well known that single-reference coupled cluster theory truncated to low orders of excitations gives the right answer for the right reason when systems are dominated by dynamical or weak correlation. Static or strong correlation is more problematic, causing often catastrophic breakdown of restricted coupled cluster. This failure can be remedied, e.g., by allowing symmetry breaking in the reference or taking a multi-reference approach, but poses an interesting theoretical problem, especially since many groups have found that simplifying the $T_{2}$ operator or the doubles amplitude equations gives better results. In singlet-paired coupled cluster, eliminating the triplet-pairing channel recovers reasonable qualitative behavior for strong correlation at the cost of a decreased description of dynamical correlation in weakly correlated situations. This behavior seems to hold for both closedand open-shell systems. In this work, we explore the coupling of the singlet- and triplet-pairing channels of $T_{2}$ and attempt to recouple them in order to recover dynamical correlation without reintroducing catastrophic failure due to strong correlation. In the weakly correlated regime, these pairing channels are only weakly coupled, and a simple recoupling gives good results. However, as strong correlation dominates, the coupling strength between the singlet- and triplet-pairing channels increases, making it difficult to perturbatively recouple the singlet- and triplet-pairing channels in this regime. Published by AIP Publishing. [http://dx.doi.org/10.1063/1.4963870]
\end{abstract}

\section{INTRODUCTION}

The electronic structure of weakly correlated systems is accurately described by the coupled cluster (CC) family of methods. ${ }^{1-4}$ Coupled cluster with single, double and perturbative triple excitations, $\operatorname{CCSD}(\mathrm{T})$, gives quantitative results for weakly correlated systems, and is routinely applied to a wide range of quantum chemical problems. Unfortunately, single-reference coupled cluster often breaks down catastrophically in the presence of strong correlation, e.g., near degeneracies in bond-breaking processes, a failure typically associated with the qualitatively incorrect nature of the mean-field reference. An archetype of this failure is in the CCSD description of the dissociation of $\mathrm{N}_{2}$ in the STO-3G basis, shown in Fig. 1, where the potential energy surface unphysically turns over around 3.2 bohrs and predicts $\mathrm{N}_{2}$ to have a very low binding energy. This failure persists even in larger bases. Despite the inadequacy of the restricted HartreeFock (RHF) reference at dissociation, recent work has shown that simplifying $\mathrm{CC}$ by eliminating or decoupling channels of the $T_{2}$ operator enables restricted single-reference CC to recover qualitatively correct behavior in describing strongly correlated systems. ${ }^{5-12}$ Thus, failures of CC to describe strong correlation can be attributed in part to instabilities in the truncated CC ansatz itself. ${ }^{13-23}$

For example, singlet-paired (triplet-paired) coupled cluster $^{12}$ is derived by retaining only the singlet-paired (triplet-paired) channel of $T_{2}$. Both singlet- and tripletpaired coupled cluster are protected from failure due to strong correlations without requiring symmetry breaking in the reference, but sacrifice some dynamical correlation. In this work, we explore the recombination of the singletand triplet-pairing channels of $T_{2}$ in order to recover weak correlation without reintroducing the failures of traditional CC for strong correlation in both closed- and open-shell systems. Although Garza et al. ${ }^{24,25}$ have had good results using density functional theory (DFT) to add dynamical correlation to singlet-paired coupled cluster, it is worth exploring the possibility of developing a full wavefunction method that treats all correlations on equal footing.

\section{THEORY AND METHODS}

We first briefly review singlet- and triplet-paired coupled cluster theory. ${ }^{12,26}$

\section{A. Restricted coupled cluster}

In order to describe singlet- and triplet-paired coupled cluster, we begin with restricted CCD. Symmetry-adapted single excitations from $T_{1}$ do not affect the singlet- and triplet pairing channels and will be added later, if needed or convenient. In closed-shell CCD, we write the wavefunction as

$$
|\Psi\rangle=e^{T_{2}}|0\rangle,
$$

where $|0\rangle$ is the RHF reference. The cluster operator $T_{2}$ is spin adapted ${ }^{27}$ and creates double excitations, 


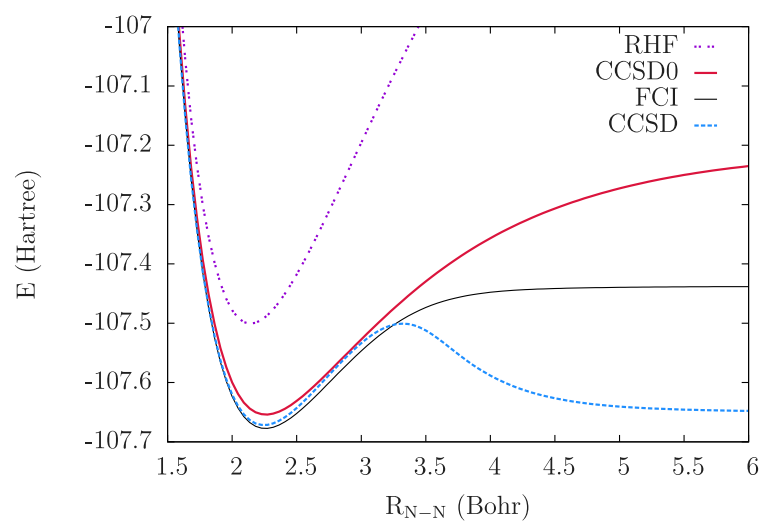

FIG. 1. $\mathrm{N}_{2}$ dissociation in STO-3G. CCSD0 recovers correct behavior while CCSD breaks down, exhibiting an unphysical bump in the potential surface and overcorrelating at dissociation. Reprinted with permission from J. Chem. Phys. 144, 244117 (2016). Copyright 2006 AIP Publishing LLC.

$$
T_{2}=\frac{1}{2} t_{i j}^{a b} c_{a \sigma}^{\dagger} c_{b \xi}^{\dagger} c_{j \xi} c_{i \sigma}
$$

where orbitals $i j(a b)$ are occupied (unoccupied) in the RHF reference, summation over repeated indices is implied, and spin indices $\sigma$ and $\xi$ are summed over up and down spins. The amplitudes $t_{i j}^{a b}$ are found by solving

$$
\left\langle{ }_{i j}^{a b}\left|e^{-T_{2}} H e^{T_{2}}\right| 0\right\rangle=R_{i j}^{a b}\left[T_{2}\right]=0,
$$

and the energy is calculated by

$$
\left\langle 0\left|e^{-T_{2}} H e^{T_{2}}\right| 0\right\rangle=E,
$$

using the amplitudes from Eq. (3). Although well-behaved when weak correlation dominates, CCD breaks down when systems take on multireference character, as evidenced by the aforementioned unphysical hump and very small binding energies in the dissociation of $\mathrm{N}_{2}$.

\section{B. Singlet- and triplet-paired coupled cluster}

Bulik et al. ${ }^{12}$ used the particle-particle/hole-hole recoupling of $T_{2}{ }^{28,29}$ to write Eq. (2) as

$$
T_{2}=T_{2}^{[0]}+T_{2}^{[1]}
$$

where

$$
\begin{gathered}
T_{2}^{[0]}=\frac{1}{2} \sigma_{i j}^{a b} P_{a b}^{\dagger} P_{i j}, \\
T_{2}^{[1]}=\frac{1}{2} \pi_{i j}^{a b} \vec{Q}_{a b}^{\dagger} \cdot \vec{Q}_{i j} .
\end{gathered}
$$

The singlet-paired operators $P_{a b}^{\dagger}$ and $P_{i j}$ give rise to $N+2$ and $N-2$ singlets, respectively. The triplet-paired operators $\vec{Q}_{a b}^{\dagger}$ and $\vec{Q}_{i j}$ give rise to $N+2$ and $N-2$ electron triplets, respectively. These pair operators are given by

$$
P_{i j}=\frac{1}{\sqrt{2}}\left(c_{j \uparrow} c_{i \downarrow}-c_{j \downarrow} c_{i \uparrow}\right)
$$

and

$$
\vec{Q}_{a b}^{\dagger} \cdot \vec{Q}_{i j}=\left(Q_{a b}^{+}\right)^{\dagger} Q_{i j}^{+}+\left(Q_{a b}^{-}\right)^{\dagger} Q_{i j}^{-}+\left(Q_{a b}^{0}\right)^{\dagger} Q_{i j}^{0}
$$

where

$$
\begin{aligned}
& Q_{i j}^{+}=c_{j \uparrow} c_{i \uparrow}, \\
& Q_{i j}^{-}=c_{j \downarrow} c_{i \downarrow}, \\
& Q_{i j}^{0}=\frac{1}{\sqrt{2}}\left(c_{j \uparrow} c_{i \downarrow}+c_{j \downarrow} c_{i \uparrow}\right) .
\end{aligned}
$$

We see from Eqs. (6) and (10) that $T_{2}^{[0]}$ and $T_{2}^{[1]}$ are, respectively, symmetric and antisymmetric upon interchange of indices $a$ and $b$ or $i$ and $j$. Thus, we have

$$
\begin{aligned}
\sigma_{i j}^{a b} & =\frac{1}{2}\left(t_{i j}^{a b}+t_{i j}^{b a}\right), \\
\pi_{i j}^{a b} & =\frac{1}{2}\left(t_{i j}^{a b}-t_{i j}^{b a}\right) .
\end{aligned}
$$

One can derive equations for $\sigma$ and $\pi$ by taking symmetric and antisymmetric combinations of the amplitude equations in Eq. (3),

$$
\begin{aligned}
& R_{i j}^{a b}\left[T_{2}\right]+R_{i j}^{b a}\left[T_{2}\right]=0, \\
& R_{i j}^{a b}\left[T_{2}\right]-R_{i j}^{b a}\left[T_{2}\right]=0
\end{aligned}
$$

and by writing $t_{i j}^{a b}=\sigma_{i j}^{a b}+\pi_{i j}^{a b}$.

We define singlet-paired CCD (CCD0) by replacing $T_{2}$ in Eq. (13) with $T_{2}^{[0]}$; similarly, we define triplet-paired CCD (CCD1) by replacing $T_{2}$ in Eq. (14) with $T_{2}^{[1]}$. As can be seen in Fig. 1, CCSD0 recovers qualitatively correct physics where standard CCSD breaks down. Including higher-order excitations with the modified $T_{2}$ operator, e.g., CCSDT0, preserves correct behavior while improving the accuracy. ${ }^{12}$

We have recently introduced a version of singlet-paired coupled cluster for open-shells: restricted open-shell singletpaired coupled cluster (ROCCSD0). ${ }^{26}$ In order to take advantage of the singlet-pairing framework, ROCCSD0 is performed on ROHF orbitals in the natural orbital basis, i.e., the basis that diagonalizes the charge density matrix. ${ }^{30}$ Working in this non-canonical framework, we then take the symmetric part of $t_{i j}^{a b}$, i.e., $\sigma_{i j}^{a b}$, in the doubly occupied to virtual block while solving for the full open-shell amplitudes, i.e., excitations into and out of the singly occupied space. ${ }^{31}$ This procedure requires symmetrizing the $o o-v v$ block of the amplitude equations. To illustrate this concept, we write the coupled cluster equations as

$$
H t=G(t),
$$

where $H t$ is shorthand for

$$
f_{i}^{k} t_{k j}^{a b}+f_{j}^{l} t_{i l}^{a b}-f_{c}^{a} t_{i j}^{c b}-f_{d}^{b} t_{i j}^{a d}
$$

and $f_{q}^{p}$ are matrix elements of the Fock operator. Spin summing Eq. (16) gives two same-spin $(\alpha \alpha$ and $\beta \beta)$ equations and one mixed-spin $(\alpha \beta)$ equation. For the $\alpha \beta$ equations, Eq. (16) becomes

$$
f_{I}^{K} t_{K \bar{J}}^{A \bar{B}}+f_{\bar{J}}^{\bar{L}} t_{I \bar{L}}^{A \bar{B}}-f_{C}^{A} t_{I \bar{J}}^{C \bar{B}}-f_{\bar{D}}^{\bar{B}} t_{I \bar{J}}^{A \bar{D}}
$$

where an overbar indicates a beta spin index, a lack of an overbar indicates an alpha spin index, and the indices run over the occupied/virtual dimensions appropriate for that spin. ROCCSD0 is defined by solving

$$
H_{\text {sym }} \sigma=G_{\text {sym }}(\sigma)
$$


in the $o o-v v$ block. $H_{\text {sym }}$ is constructed by replacing the Fock matrices in Eq. (17) by Fock charge,

$$
\mathcal{F}=\frac{1}{2}\left(f^{\alpha}+f^{\beta}\right) \text {. }
$$

We have found that this method remedies the failures of standard ROCCSD to describe static correlation in open-shell systems in a manner similar to that seen for closed-shell CCSD0: it is protected from failure, but sacrifices part of the description of dynamical correlation. ${ }^{26}$

\section{Recoupling the singlet- and triplet-pairing channels}

The lack of quantitative accuracy in the singlet-paired and triplet-paired coupled cluster models described above is apparent in the structure of the singlet-pair operator in Eq. (8), where there is clearly no explicit correlation between same-spin electrons, and in the high energies CCSD1 predicts for the dissociation limit of $\mathrm{N}_{2}$ in Fig. 1 .

The coupling between the singlet- and triplet-pairing channels of $T_{2}$ is needed for the exact answer. "Full" CC0 including all excitations, but using $T_{2}^{[0]}$ to describe the doubles, would not be correct. Yet, this same coupling is apparently responsible for the breakdown of truncated coupled cluster in the presence of strong correlation. Here, we explore the possibility of allowing $\sigma$ and $\pi$ to couple in order to recover dynamical correlation without reintroducing the breakdown due to static correlation. One way to do this would be first to solve the CCD0 equations, obtaining a set of $\sigma_{i j}^{a b}$ amplitudes. We could then solve for a $\pi_{i j}^{\prime a b}$ correction, giving a set of full $t_{i j}^{a b}$ amplitudes subject to the constraint that the $\sigma_{i j}^{a b}$ amplitudes from the CCD0 calculation remain fixed or "frozen." Likewise, we could first solve for a set of $\pi_{i j}^{a b}$ amplitudes from the CCD1 equations, which we then freeze, calculating a $\sigma_{i j}^{\prime a b}$ correction to construct full $t_{i j}^{a b}$ amplitudes. We refer to these procedures as frozen sigma (FSig) and frozen pi (FPi) CCD, respectively. Singlet- and triplet-paired coupled cluster can be obtained as zeroth order approximations to full CC. FSig and FPi include first-order corrections to the missing singlet-triplet coupling. This series should sum to standard $\mathrm{CC}$, which we know breaks down, but we want to know whether we can make low-order corrections to $\mathrm{CC} 0$ and $\mathrm{CC} 1$ in order to recover the correlations we miss in the zeroth-order methods without reintroducing the failures of coupled cluster theory.

In order to include single excitations, constructing FSigCCSD and FPiCCSD, we must decide whether to allow the singles to relax due to the $\pi_{i j}^{\prime a b}$ and $\sigma_{i j}^{\prime a b}$ corrections following the initial CCSD0 or CCSD1 calculation, or whether we will freeze them as well. We have found that allowing the single excitations to relax during the second part of an FSigCCSD or FPiCCSD calculation provides slightly better energies, so we have used this approach. Although we can in principle include higher-order excitations, we have not done so here.

The open-shell equivalent of FSigCCSD is only slightly more complicated. Since ROCCSD0 generates open-shell $t_{i j}^{a b}$ amplitudes, we must decide whether to freeze them as well or allow them to relax when calculating the $\pi_{i j}^{\prime a b}$ correction. We employ the former approach, having found that allowing the open-shell $t_{i j}^{a b}$ amplitudes to relax in response to the antisymmetric $\pi_{i j}^{a b}$ correction in FSigCCSD significantly reintroduces the pathological breakdown of standard ROCCSD.

\section{Computational details}

All post-SCF calculations with modified $T_{2}$ were performed using in-house code. Hartree-Fock and standard coupled-cluster calculations were done in Gaussian 09. ${ }^{32}$ In order to maximize strong-correlation effects, we work in small basis sets throughout. ${ }^{33,34}$ Strong correlation is much less basis set dependent than dynamical correlation and should dominate in small bases. Our in-house code uses Cartesian $d$ functions and does not support point-group symmetry. We show coupled cluster results for multiple SCF references where we found more than one smooth SCF dissociation curve. Lastly, we use DIIS to accelerate convergence of our coupled cluster methods. ${ }^{35}$ Our coupled cluster calculations with modified $T_{2}$ typically converge in a number of iterations comparable to standard coupled cluster, but can become more difficult to converge at stretched bond lengths.

\section{RESULTS}

\section{A. Linear $\mathrm{H}_{6}$}

Figure 2 shows the results of dissociating a linear chain of six equally spaced hydrogen atoms in the ccpVDZ basis, ${ }^{36}$ using various coupled cluster models. For this system, the breakdown of standard coupled cluster is apparent, with CCSD turning over at around 3.6 bohrs. Both FPiCCSD and FSigCCSD recover correlation at equilibrium, giving values comparable to those given by unrestricted $\operatorname{CCSD}(\mathrm{T})$. UCCSD(T) should be quite accurate here, but requires significant spin contamination in the underlying UHF wavefunction, and we would like to preserve symmetries of the Hamiltonian as much as possible. In general, $\mathrm{CC} 0$ already

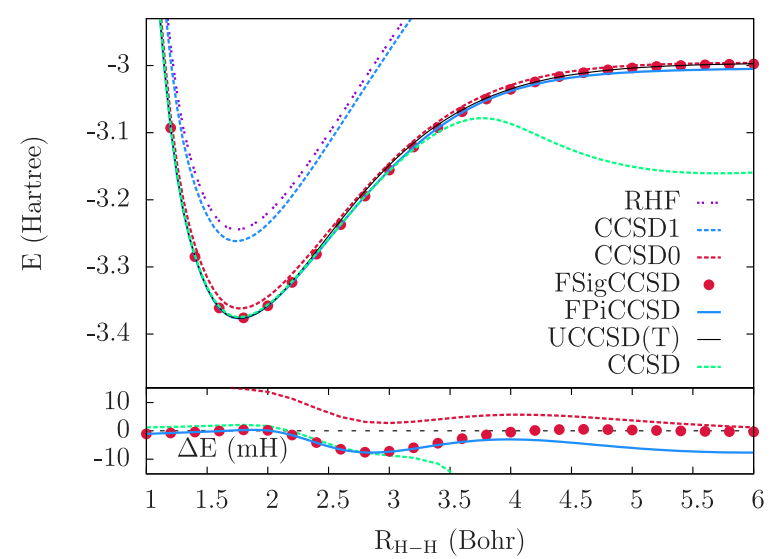

FIG. 2. $\mathrm{H}_{6}$ dissociation in cc-pVDZ. CCSD breaks down, turning over at around 3.5 bohrs. CCSD0 and CCSD1 recover correct behavior. FSigCCSD and FPiCCSD recover dynamical correlation without reintroducing the breakdown of standard CC. 
does pretty well for systems where we basically localize one electron per site, e.g., hydrogen clusters. While this statement holds here, FSigCCSD recovers more correlation than CCSD0 throughout the range of bond lengths. While CCSD1 is quite poor, adding the $\sigma_{i j}^{\prime a b}$ correction recovers excellent energies while preserving good behavior of the energy surface.

\section{B. Circular $\mathrm{H}_{4}$}

$\mathrm{H}_{4}$ on a circle is known to cause problems for standard CC. Here, the system consists of four Hydrogen atoms on a circle of radius $R=1.738 \AA$, separated by an angle $\theta$. For small and large $\theta$, the system is approximately two non-interacting $\mathrm{H}_{2}$ molecules. As $\theta$ approaches $90^{\circ}$, the system acquires a two-fold degeneracy. The molecule is shown graphically in Fig. 3.

The top plot of Fig. 4 shows results from CCD and related methods for this system in Dunning's DZP basis $^{37,38}$ for $\theta$ ranging from $85^{\circ}$ to $95^{\circ}$. The bottom plot of Fig. 4 shows the same calculations, with the addition of singles. Full configuration interaction shows a smooth curve with a local maximum at $\theta=90^{\circ} .{ }^{39}$ The breakdown of standard coupled cluster for this system consists of a deep minimum at $\theta=90$ and a cusp in the curve, due to the crossing of two SCF solutions. Adding singles does not remedy the deficiencies of $\mathrm{CCD}$, but exacerbates both the depth of the minimum and the sharpness of the cusp. In the top plot of Fig. 4, both CCD0 and CCD 1 correctly predict a local maximum at $\theta=90^{\circ}$, but still give an unphysical cusp (the CCD1 energy for this system is too high to be shown on this plot). Frozen-Sigma CCD improves noticeably on $\mathrm{CCD} 0$, shifting the energy toward the FCI result, and slightly smoothing out the cusp. Frozen-Pi CCD improves on CCD1 by shifting the energy down, but, while the recoupling of $\sigma_{i j}^{a b}$ and $\pi_{i j}^{a b}$ smooths the cusp at $\theta=90$, here it reintroduces a slight local minimum. The top plot of Fig. 4 also shows results for $\operatorname{CCD}(0+1)$, which means we take the correlation energy to be simply the sum of the CCD0 and CCD1 correlation energies. There is no coupling between the singlet- and triplet-pairing channels in $\mathrm{CCD}(0+1)$. Although this procedure overcorrelates in

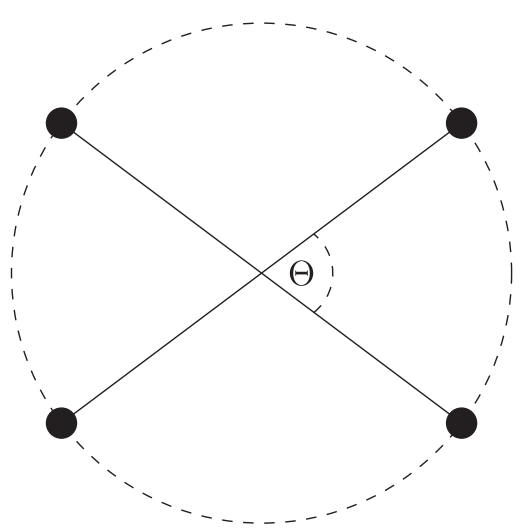

FIG. 3. Geometry of $\mathrm{H}_{4}$ on a circle. Adapted with permission from I. W. Bulik et al. J. Chem. Theory Comput. 11, 3171 (2015). Copyright 2015 American Chemical Society.
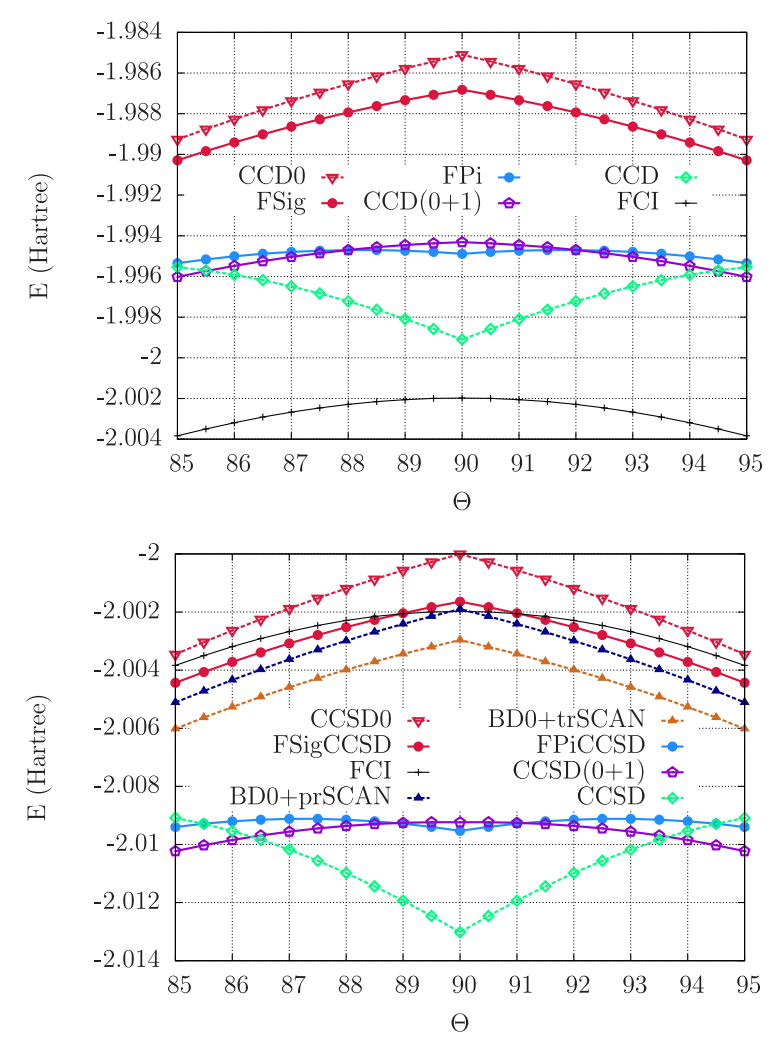

FIG. 4. $\mathrm{H}_{4}$ circle in Dunning's DZP basis $(4 \mathrm{~s} 1 \mathrm{p} / 2 \mathrm{~s} 1 \mathrm{p})$ using coupled cluster models with doubles (top) and singles and doubles (bottom). Standard coupled cluster breaks down, predicting a deep minimum and cusp at $\theta=90^{\circ}$. $\mathrm{CC} 0$ and $\mathrm{CC} 1$ models recover a maximum, but still have a cusp. FSig recovers correlation. FPi recovers correlation but reintroduces the erroneous local minimum. $\mathrm{CC}(0+1)$ recovers a smooth curve.

general, here the model gives surprising results. Not only does $\mathrm{CCD}(0+1)$ give reasonable energies for this system, but it also gives correct physical behavior, eliminating the unphysical cusp given by the other coupled-cluster methods. We would expect to need connected quadruple excitations to accurately capture this feature, but apparently for this system, the necessary quadruples are factorizable into doubles.

The bottom plot in Fig. 4 shows the result of adding singles to the calculations shown in the top plot of Fig. 4 . We see the same general trends as for the doubles-only calculations. Although CCSD0 and FSigCCSD happen to be near FCI, and all the methods recover some correct behavior relative to CCSD, the methods generally over-correlate. We also show BD0+prSCAN and BD0+trSCAN results from Ref. 25. Briefly, these methods combine the singlet-paired correlation energy from a singlet-paired Brueckner doubles (BD0) with the parallel-spin correlation $(\mathrm{BD} 0+$ prSCAN) and triplet-paired correlation energy (BD0+trSCAN) obtained from the strongly constrained and appropriately normed ${ }^{40}$ (SCAN) meta-GGA functional. The standalone BD0 results are comparable to CCSD0 for this system. Singlet-paired coupled cluster plus DFT models have recently been successfully applied to a variety of systems, ${ }^{24,25}$ and here, FSigCCSD is comparable to the BD0+DFT methods, even overcorrelating less throughout the potential energy surface. 
To get an idea of basis set effects, we show results of the above calculations on $\mathrm{H}_{4}$ in the cc-pVTZ basis ${ }^{36}$ in Fig. 5. In the top plot of Fig. 5, we see that the larger basis decreases the depth of the minimum predicted by CCD, but the erroneous minimum and unphysical cusp persist. In the top plot of Fig. 5, FPiCCD no longer exhibits the unphysical cusp at $\theta=90^{\circ}$. It is curious that FPiCCD and $\mathrm{CCD}(0+1)$ give essentially the same result near $\theta=90$ here, since in $\operatorname{CCD}(0+1)$ there is no coupling between the singlet- and triplet-pairing channels. This result is not due to CCD1 giving zero correlation energy; CCD1 gives correlation energies of about 0.01 Hartree across the range of $\theta$ shown here. Rather, in this case the FPiCCD $\sigma_{i j}^{\prime a b}$ correction to CCD1 returns nearly the same correlation energy as CCD0, despite the $\pi_{i j}^{a b}$ contribution to FPiCCD being non-zero. The result of adding singles is shown in the bottom plot of Fig. 5. The minimum predicted by standard $\mathrm{CC}$ becomes deeper, and FPiCCSD reverts to predicting a slight local minimum at $\theta=90$. There is now less similarity between FPiCCSD and $\operatorname{CCSD}(0+1)$. We note that, when we perform FSigCCSD and FPiCCSD calculations, we allow the singles amplitudes to relax due to the correction to the $t_{i j}^{a b}$ amplitudes. Separate CCSD0 and CCSD1 calculations might result in substantially different singles amplitudes, worsening the description given by $\operatorname{CCSD}(0+1)$.
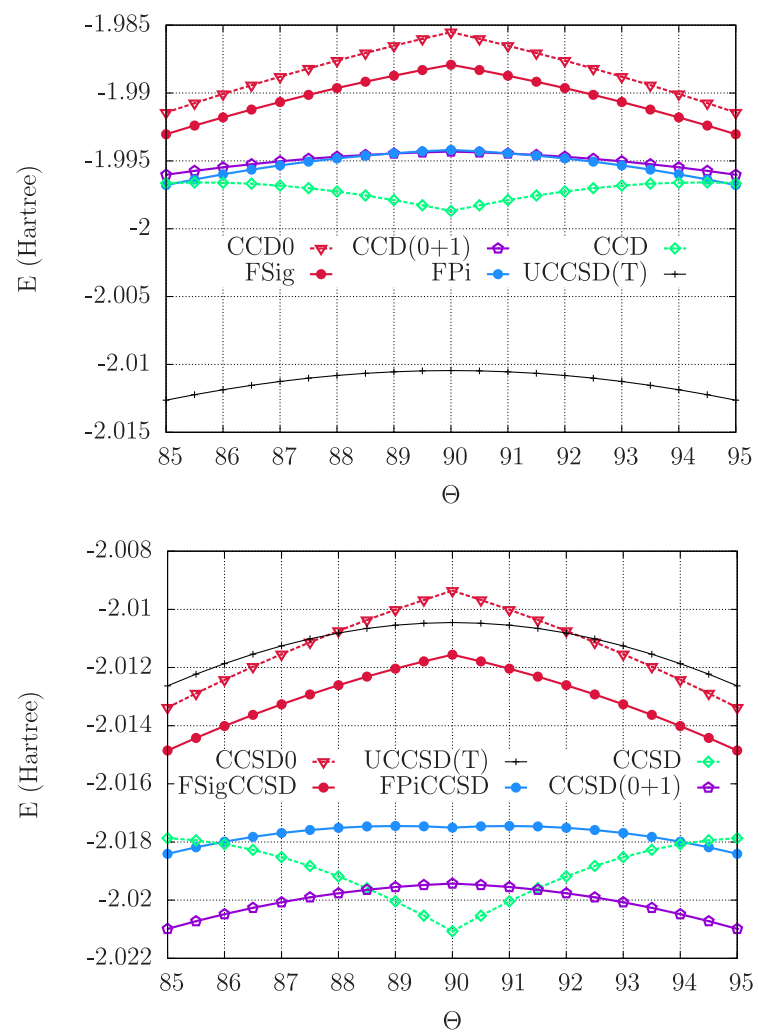

FIG. 5. $\mathrm{H}_{4}$ circle in cc-pVTZ using coupled cluster models with doubles (top) and singles and doubles (bottom). Standard coupled cluster breaks down, predicting a deep minimum and cusp at $\theta=90^{\circ}$. CC0 and CC1 models recover a maximum, but still have a cusp. FSig recovers correlation. FPiCCD recovers correlation and smooths out the cusp. FPiCCSD recovers correlation, but reintroduces the erroneous local minimum. $\mathrm{CC}(0+1)$ recovers a smooth curve. In general, the larger basis set results in larger overcorrelation by the FSig and FPi methods.

\section{C. $\mathrm{N}_{2}$}

The top plot of Fig. 6 shows the dissociation of $\mathrm{N}_{2}$ in the cc-pVDZ basis. ${ }^{36}$ Near equilibrium, FSigCCD and FPiCCD energies are not only better than both CCD0 and CCD1, they are better than standard CCD. At dissociation, where the singlet-triplet coupling becomes problematic, FPiCCD and FSigCCD split the difference between CCD0 and CCD1. This means that, although we improve our description of the system at dissociation by adding a $\sigma_{i j}^{\prime a b}$ correction to CCD1, adding the $\pi_{i j}^{\prime a b}$ correction to CCD0 worsens the result. We compare to long range-corrected $\mathrm{CCD} 0+\operatorname{trSCAN}$ from Ref. 25, which recovers significant correlation from standard CCD, but overcorrelates and reintroduces the hump in the energy surface. For standard CCD, the breakdown can be attributed to the correlation energies from the singletand triplet-pairing channels of $T_{2}$ diverging in opposite directions. That is, as $\mathrm{N}_{2}$ is dissociated, the singlet-paired amplitudes give increasingly negative correlation energy, while the triplet-paired component of the correlation energy becomes increasingly positive. Although CCD dissociates $\mathrm{N}_{2}$ poorly, the result is only as good as it is because the singlet and triplet-paired correlation energies cancel to some extent. This cancellation effect is minimized for FSig and
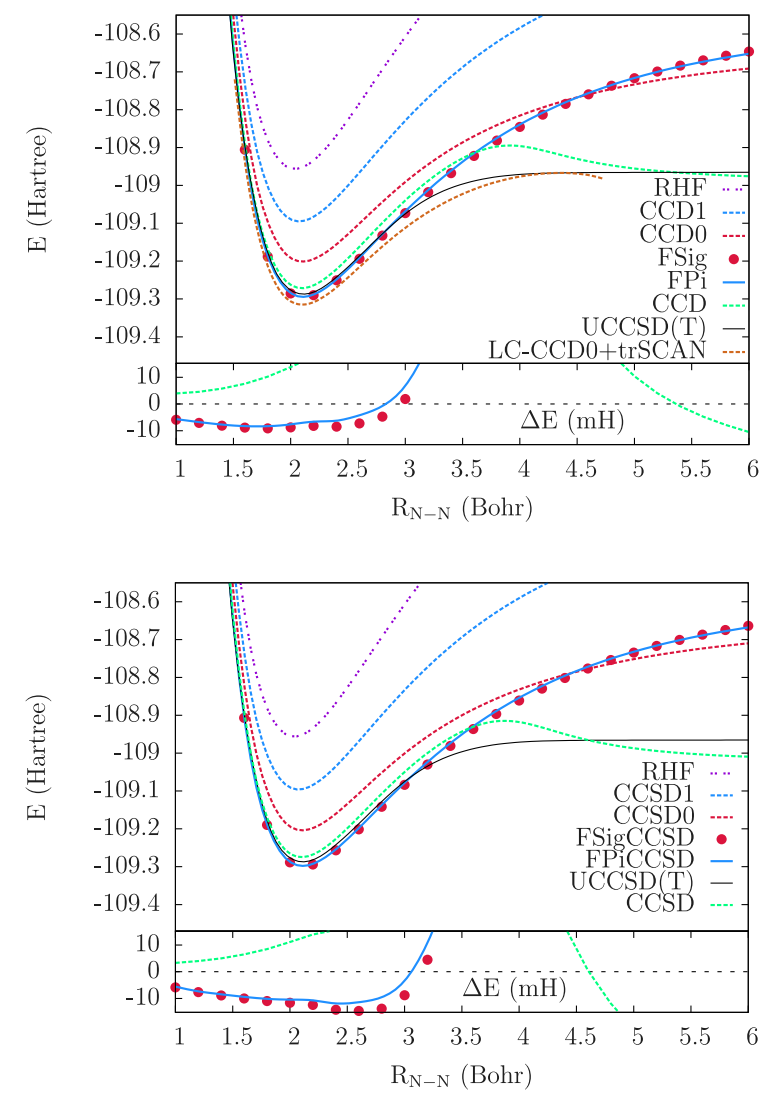

FIG. 6. $\mathrm{N}_{2}$ dissociation in cc-pVDZ using various coupled cluster models with doubles (top) and singles and doubles (bottom). CCSD unphysically turns over around 3.6 bohrs. CCD0 and CCD1 recover correct behavior. FSigCCD recovers correlation at equilibrium, but increases the energy at dissociation relative to $\mathrm{CC} 0$. FPiCCD recovers correlation across the curve, giving results nearly identical to FSigCCD. Adding singles has little effect on the energy surfaces, but highlights that FSigCCSD and FPiCSCD probably overcorrelate near equilibrium. 
FPi, due to the weak coupling between the singlet- and triplet-pairing channels. The bottom plot of Fig. 6 shows the same calculations, with the addition of singles. Adding singles makes the overcorrelation of standard coupled cluster more pronounced, but seems to have little effect on the modified coupled cluster models. Although the energies are shifted downward slightly at dissociation, the results are generally the same, including the near identical energies given by FSigCCSD and FPiCCSD.

\section{Triplet $\mathbf{N}_{2}$}

The top plot of Fig. 7 shows the dissociation of triplet $\mathrm{N}_{2}$ relative to the energies of the quartet and doublet nitrogen atoms in the cc-pVDZ basis. We found two ROHF curves for this system. Reference $A$ is lower at equilibrium, while reference $B$ dissociates to a lower limit. ROCCSD-B is well behaved and gives excellent results, while ROCCSD-A fails dramatically, turning over at around 3.8 bohrs. ROCCSD0-A dissociates to nearly the correct limit, while ROCCSD-B maintains good behavior, but part of the weak correlation. Although both FSigROCCSD curves go to too low of a limit, they give much more reasonable results at dissociation than standard coupled cluster on reference $A$, in a sense alleviating the problem of ROHF reference choice.

The bottom plot of Fig. 7 shows total energies for the dissociation of triplet $\mathrm{N} 2$, highlighting the effect of the $\pi_{i j}^{\prime a b}$ correction in the FSigCCSD calculation. ROCCSD0 on both curves is well behaved, but sacrifices a substantial amount
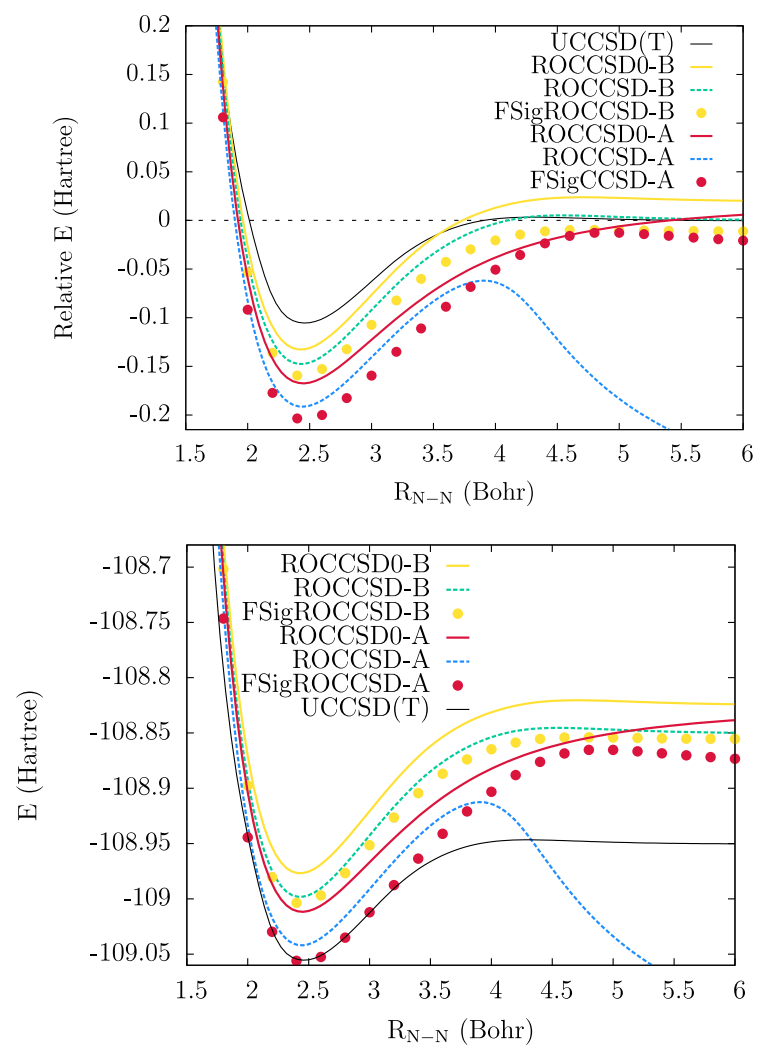

FIG. 7. Dissociation of triplet $\mathrm{N}_{2}$ in cc-pVDZ. FSigROCCSD on both SCF references improves on ROCCSD0 throughout the range of bond lengths, especially at equilibrium. of correlation throughout the energy surface. We are able to recover dynamical correlation using FSigROCCSD on both references. FSigROCCSD-A does have a minor bump around 4.6 bohrs. This hint of the CCSD-like breakdown is worsened if we allow the open-shell amplitudes to relax in response to $\pi_{i j}^{\prime a b}$. This result is reminiscent of that seen for frozen-pair coupled cluster. ${ }^{10}$ There, freezing the paired $T_{2}$ amplitudes and calculating the non-paired correction reintroduced a hump in the dissociation curve of singlet $\mathrm{N}_{2}$. However, we can attribute the unphysicality of frozen-pair coupled cluster to the coupling of the singlet- and triplet-pairing channels that occurs in the non-paired block of the $T_{2}$ amplitude equations. We have broken the direct coupling between $\sigma_{i j}^{a b}$ and $\pi_{i j}^{a b}$ here, but as with the circular $\mathrm{H}_{4}$ case, calculating the $\pi_{i j}^{\prime a b}$ contribution from frozen $\sigma_{i j}^{a b}$ amplitudes begins to reintroduce the breakdown of standard coupled cluster.

\section{DISCUSSION}

The recoupling of the singlet- and triplet-pairing channels of $T_{2}$ is ostensibly partly responsible for the breakdown of truncated, single-reference restricted coupled cluster in the presence of strong correlation. Decoupling these pairing channels in singlet- and triplet-paired coupled cluster recovers more correct behavior, but sacrifices part of the description of dynamical correlation. We have explored recoupling the singlet- and triplet-pairing channels of $T_{2}$ via frozen-sigma and frozen-pi coupled cluster in both closed and open shells in order to recover dynamical correlation without allowing and reintroducing the breakdown of standard coupled cluster. When standard coupled cluster gives a good description of the system, e.g., molecules near equilibrium, FSigCC and FPiCC give results comparable to standard coupled cluster. For strongly correlated situations, however, the quality of the FSigCC and FPiCC results depends on the nature of the correlations present. For the circular four-hydrogen cluster, FSigCC and FPiCC recover correlation from $\mathrm{CC} 0$ and $\mathrm{CC} 1$. However, FSigCC at dissociation for $\mathrm{N}_{2}$ goes to a higher limit than $\mathrm{CC} 0$. This result tells us that the way in which the coupling between the singlet- and triplet-pairing channels of $T_{2}$ causes the breakdown of standard CC may be systemspecific as well, and a good description may require a more complicated treatment, e.g., recoupling higher-order excitations. For open-shell FSigCCSD calculations, we found it useful to freeze open-shell $t_{i j}^{a b}$ amplitudes in addition to $\sigma_{i j}^{a b}$. Allowing the open-shell $t_{i j}^{a b}$ amplitudes to relax in the presence of the $\pi_{i j}^{\prime a b}$ correction significantly reintroduces the breakdown of standard coupled cluster. We speculate that for open shells the indirect coupling of $\sigma$ and $\pi$ through the open-shell amplitude blocks contributes to the breakdown of standard ROCCSD for strong correlation, not just the direct coupling of the singlet- and triplet-pairing channels in the doubly occupied to virtual block of $T_{2}$.

FSig and FPi can be viewed as first-order corrections to $\mathrm{CC} 0$ and $\mathrm{CC} 1$, respectively. We can imagine resumming this perturbative expansion by first calculating $\sigma$ amplitudes from $\mathrm{CC} 0$, then calculating a $\pi^{\prime}$ correction while keeping $\sigma$ fixed, and then calculating a new $\sigma^{\prime}$ keeping $\pi^{\prime}$ frozen, and 
so on. However, early tests of this idea did not appear to be meaningful.

Returning to the CC0+DFT formalism of Garza et al., ${ }^{25}$ the CCD0+tDFT energy is given by

$$
E_{C}^{\mathrm{CCD} 0+\mathrm{tDFT}}=E_{C}^{\mathrm{CCD} 0}+3 E_{c \uparrow \uparrow}^{D F A}\left[n_{\uparrow}, n \downarrow\right],
$$

where the " $\mathrm{t}$ " in tDFT indicates that we take into account the full triplet-paired contribution to $T_{2}$. We see that the wavefunction equivalent of the $\mathrm{CC} 0+\mathrm{tDFT}$ energy expression is

$$
\begin{aligned}
E_{C}= & E_{C}^{\alpha \beta}[\sigma(\mathrm{CC} 0)]+E_{C}^{\alpha \beta}[\pi(\mathrm{CC})] \\
& +E_{C}^{\alpha \alpha}[\pi(\mathrm{CC})]+E_{C}^{\beta \beta}[\pi(\mathrm{CC})],
\end{aligned}
$$

that is, the singlet-paired component of the energy comes from $\mathrm{CC} 0$, while the triplet-paired component of the energy comes from standard coupled cluster. Expression (20) has been quite useful for calculating both energies and properties, ${ }^{24,25}$ but Eq. (21) would break down since the triplet-paired component of the correlation energy from standard coupled cluster is poorly behaved in the presence of strong correlation.

\section{CONCLUDING REMARKS}

We asked whether a first-order recoupling of the singletand triplet-pairing channels of $T_{2}$ might recover dynamical correlation without breaking down due to strong correlation. Although not as generally accurate as we might have hoped, the successes and failures of FSigCC and FPiCC tell us something about the relationship between the strength of the coupling between the singlet- and triplet-pairing channels and the strength of the correlations that characterize the system. When systems are weakly correlated, FSigCC and FPiCC are quite accurate, indicating that the singlet-triplet coupling in these cases is also weak, and a low-order recoupling is sufficient. We have seen this hold for molecules at equilibrium as well as stretched $\mathrm{H}_{6}$, a not extremely strongly correlated system. When systems are strongly correlated, however, such as the problem of stretched $\mathrm{N}_{2}$, FSigCC and FPiCC are inaccurate and in some cases give unexpectedly poor results. This observation would seem to indicate that for strong correlation, the singlet-triplet coupling becomes more complex, and therefore, a first-order recoupling of these terms is inadequate. This conclusion is not surprising, since it is generally understood that an accurate description of $N$ strongly correlated electrons requires the presence of up through $T_{N}$ when working within the exponential ansatz of coupled cluster theory. Recent work suggests that lower-order truncations are possible if the exponential ansatz of coupled cluster theory is abandoned. ${ }^{23}$ Work along the lines of including these recouplings to all orders using symmetry projection ideas via a sinh polynomial, rather than an exponential, is presented elsewhere. $^{41}$

\section{ACKNOWLEDGMENTS}

We thank Alejandro Garza for providing the $\mathrm{CC} 0+\mathrm{DFT}$ data. This material is based on work supported by the U.S. Department of Energy Heavy Element Chemistry program (Grant No. DE-FG02-04ER15523). G.E.S. is a Welch Foundation Chair (Grant No. C-0036). J.A.G. gratefully acknowledges support of the National Science Foundation Graduate Research Fellowship Program (Grant No. DGE1450681).

${ }^{1}$ J. Paldus and X. Li, "A critical assessment of coupled cluster method in quantum chemistry," in Advances in Chemical Physics Volume 110, edited by I. Prigogine and S. A. Rice (John Wiley \& Sons, Inc., Hoboken, NJ, USA, 1999).

${ }^{2}$ T. D. Crawford and H. F. Schaefer, "An introduction to coupled cluster theory for computational chemists," in Reviews in Computational Chemistry (John Wiley \& Sons, Inc., 2007), pp. 33-136.

${ }^{3}$ R. J. Bartlett and M. Musial, Rev. Mod. Phys. 79, 291 (2007).

${ }^{4}$ I. Shavitt and R. J. Bartlett, Many-Body Methods in Chemistry and Physics (Cambridge University Press, New York, 2009).

${ }^{5}$ P. A. Limacher, P. W. Ayers, P. A. Johnson, S. de Baerdemacker, D. Van Neck, and P. Bultinck, J. Chem. Theor. Comput. 9, 1394 (2013).

${ }^{6}$ P. A. Limacher, T. D. Kim, P. W. Ayers, P. A. Johnson, S. de Baerdemacker, D. Van Neck, and P. Bultinck, Mol. Phys. 112, 853 (2014).

${ }^{7}$ P. Tecmer, K. Boguslawski, P. A. Johnson, P. A. Limacher, M. Chan, T. Verstraelen, and P. W. Ayers, J. Phys. Chem. A 118, 9058 (2014).

${ }^{8}$ K. Boguslawski, P. Tecmer, P. W. Ayers, P. Bultinck, S. De Baerdemacker, and D. Van Neck, Phys. Rev. B 89, 201106(R) (2014).

${ }^{9}$ T. Stein, T. M. Henderson, and G. E. Scuseria, J. Chem. Phys 140, 214113 (2014).

${ }^{10}$ T. M. Henderson, I. W. Bulik, T. Stein, and G. E. Scuseria, J. Chem. Phys. 141, 244104 (2014).

${ }^{11}$ T. M. Henderson, I. W. Bulik, and G. E. Scuseria, J. Chem. Phys. 142, 214116 (2015).

${ }^{12}$ I. W. Bulik, T. M. Henderson, and G. E. Scuseria, J. Chem. Theor. Comput. 11, 3171 (2015).

${ }^{13}$ J. Paldus, J. Č́ížek, and M. Takahashi, Phys. Rev. A 30, 2193 (1984).

${ }^{14}$ P. Piecuch and J. Paldus, Theor. Chim. Acta 78, 65 (1990).

${ }^{15}$ P. Piecuch, R. Toboła, and J. Paldus, Phys. Rev. A. 54, 1210 (1996).

${ }^{16}$ P. Piecuch, S. A. Kucharski, and K. Kowalski, Chem. Phys. Lett. 344, 176 (2001).

${ }^{17}$ R. J. Bartlett and M. Musial, J. Chem. Phys. 125, 204105 (2006).

${ }^{18}$ D. W. Small and M. Head-Gordon, J. Chem. Phys. 137, 114103 (2012).

${ }^{19}$ D. Katz and F. R. Manby, J. Chem. Phys. 139, 021102 (2013).

${ }^{20}$ D. Katz, J. Chem. Phys. 141, 061101 (2014).

${ }^{21}$ D. Katz, D. Kreplin, H. Werner, and F. R. Manby, J. Chem. Phys. 142, 064111 (2015).

${ }^{22}$ V. Rishi, A. Perera, and R. J. Bartlett, J. Chem. Phys. 144, 124117 (2016).

${ }^{23}$ M. Degroote, T. M. Henderson, J. Zhao, J. Dukelsky, and G. E. Scuseria, Phys. Rev. B 79, 125124 (2016).

${ }^{24}$ A. J. Garza, A. G. S. Alencar, and G. E. Scuseria, J. Chem. Phys. 143, 244106 (2015).

${ }^{25}$ A. J. Garza, I. W. Bulik, A. G. S. Alencar, J. Sun, J. P. Perdew, and G. E. Scuseria, Mol. Phys. 114, 997 (2015).

${ }^{26}$ J. A. Gomez, T. M. Henderson, and G. E. Scuseria, J. Chem. Phys. 144, 244117 (2016).

${ }^{27}$ G. E. Scuseria, C. L. Janssen, and H. F. Schaefer III, J. Chem. Phys. 89, 7382 (1989).

${ }^{28}$ J. Paldus, J. Chem. Phys. 67, 303 (1977).

${ }^{29}$ J. Paldus, B. G. Adams, and J. Cizek, Int. J. Quantum Chem. 11, 813 (1977).

${ }^{30}$ A. Szabo and N. S. Ostlund, Modern Quantum Chemistry (McGraw-Hill, Inc., New York, 1989).

${ }^{31}$ G. E. Scuseria, Chem. Phys. Lett. 176, 27 (1991).

${ }^{32}$ M. Frisch, G. W. Trucks, H. B. Schlegel, G. E. Scuseria et al., Gaussian 09, Revision D.01, Gaussian, Inc., Wallingford, CT, 2013..

${ }^{33}$ D. J. Feller, J. Comput. Chem. 17, 1571 (1996).

${ }^{34}$ K. L. Schuchardt, B. T. Didier, T. Elsethagen, L. Sun, V. Gurumoorthi, J. Chase, J. Li, and T. L. Windus, J. Chem. Inf. Model. 47, 1045 (2007).

${ }^{35}$ G. E. Scuseria, T. J. Lee, and H. F. Schaefer, Chem. Phys. Lett. 130, 236 (1986).

${ }^{36}$ T. H. Dunning, J. Chem. Phys. 90, 1070 (1989).

${ }^{37}$ T. H. Dunning, Jr., J. Chem. Phys. 52, 2823 (1970).

${ }^{38}$ T. H. Dunning, Jr. and P. J. Hay, in Methods of Electronic Structure Theory, edited by H. F. Schaefer III (Plenum Press, New York, 1977), Vol. 3.

${ }^{39}$ T. Van Voorhis and M. Head-Gordon, J. Chem. Phys. 113, 8873 (2000).

${ }^{40}$ J. Sun, A. Ruzsinszky, and J. P. Perdew, Phys. Rev. Lett. 115, 036402 (2015).

${ }^{41}$ Y. Qiu, T. M. Henderson, and G. E. Scuseria, J. Chem. Phys. 145, 111102 (2016). 